REPORTS OF MORPHOLOGY
Official Journal of the Scientific Society of Anatomists,
Histologists, Embryologists and Topographic Anatomists
of Ukraine
journal homepage: https://morphology-journal.com

\title{
Correlations of linear sizes of molars with cephalometric indicators of practically healthy men of the northern region of Ukraine
}

Polishchuk S.S., Kotsyura O.O., Dmitriev M.O., Orlovskiy V.O., Popov M.V. National Pirogov Memorial Medical University, Vinnytsya, Ukraine

\section{ARTICLE INFO}

Received: 18 December, 2019

Accepted: 22 January, 2020

UDC: $611.314: 616.714 .1-071.3: 79-$ 055.1(477)

\section{CORRESPONDING AUTHOR}

e-mail: olyatkokotsyura@gmail.com Kotsyura O.O.

\begin{abstract}
The question of the relationship between the metric characteristics of cranial structures and odontometric parameters, and the possibility of building models based on them, which can later find their practical application in dental practice, remain a pressing problem of modern medical science. The purpose of the study is to establish the features of the relationship between the linear dimensions of molars with the cephalometric parameters of the brain and facial skull of practically healthy men in the northern region of Ukraine. 32 practically healthy residents of the northern region of Ukraine (from Kyiv, Zhytomyr, Sumy and Chernihiv regions) underwent computed tomography followed by odontometry of molars and cephalometry. The correlations between odontometric and cephalometric parameters were assessed using Spearman's nonparametric statistics in the licensed package "Statistica 6.1". The peculiarities of the correlations of the studied characteristics of molars with the indicators of the cerebral skull are established - multiple, only direct reliable $(r=0.35-0.57)$ and unreliable $(r=0.30-0.35)$ average correlation force (18.7\% on the upper jaw, of which the relative majority with vestibular-lingual and mesio-distal dimensions and $24.1 \%$ on the lower jaw, of which the relative majority with the height of the teeth, their crowns and the length of the tooth roots); as well as with the indicators of the facial skull - mostly direct reliable ( $r=0.35-0.51)$ and unreliable medium strength $(r=0.30-0.37)$ correlations, in most cases of a single nature $(6.0 \%$ on the upper jaw and $6.6 \%$ on the lower jaw, almost uniformly with the height of the teeth, their crowns, root length, vestibular-lingual and mesio-distal dimensions). The results of correlation analysis of craniofacial structures are necessary for the correct construction of mathematical models, which can later find their practical application in dental practice and forensic medicine.

Keywords: correlations, cephalometry, odontometry, administrative-territorial regions of Ukraine.
\end{abstract}

\section{Introduction}

Throughout its existence, mankind has sought to understand the basic principles of building the world and the phenomena observed in it. And naturally, the question of human anatomy and physiology and the search for an understanding of the laws of its structure and related functions, have occupied and occupy one of the leading places in world scientific research. With the beginning of the digital age and the creation of the artificial intelligence system, with the improvement of statistical analysis tools, mankind has the opportunity to calculate and analyze large data sets. And this state of affairs has forced us to reconsider and rethink things that lie on the surface - the relationship of various functionally connected anatomical components. Morphologists, like no other today, can provide new information on understanding the basic principles of normal anatomy, the relationship between the shape and function of the various structural components of the human body. And in dentistry, as never before, the issues of modeling and reproduction of the structures of the dental apparatus remain acute. With powerful computational and statistical tools, specialists in many specialties related to the work with the craniofacial complex return to the question of studying the anatomical norm. The biggest number of questions arises about dental structures. Their anatomical diversity and complex morphofunctional connections with craniofacial structures provide a favorable basis for such studies to a greater extent in the fields of anthropology, dentistry and forensic medicine $[3,6,9,10]$. It should be 
noted that a large number of modern researchers in the study of the main odontological normative indicators indicate the need to consider ethnicity and gender [12, 15, 17, 22].

The main function of the dental apparatus is to grind food and prepare it for further digestion. This function is performed by the masticatory group of teeth, and that is why the molars were given the status of a large occlusion key. No wonder a large number of modern scientific papers are devoted to the study of the relationship of the masticatory group of teeth with other cranial structures, which also indicate the importance of considering the ethnic and sexual specifications of man [5, 13, 20, 21].

The question of the relationship between the metric characteristics of cranial structures and odontometric parameters, and the possibility of building models based on them, which can later find their practical application in dental practice, has been raised by scientists more than once $[4,7,8,10]$, because such models can be used by physicians not only for an individual approach to each patient but also for screening mass examinations [11] in order to predict the occurrence of a pathology, and thus create risk groups that will require special dental supervision in the future.

That is why the purpose of our study is to establish the features of the relationship between the linear dimensions of molars with the cephalometric parameters of the brain and facial skull of practically healthy men in the northern region of Ukraine.

\section{Materials and methods}

For the study, 32 residents of the northern region of Ukraine from Kyiv, Zhytomyr, Sumy and Chernihiv regions were selected, with favorable, moderately favorable and satisfactory ecological living conditions. Computed tomography was performed using a Veraviewepocs-3D dental cone tomography scanner (Morita, Japan). Committee on Bioethics of National Pirogov Memorial Medical University, Vinnytsya established (Minutes №19 from 08.11.2012, Minutes №5 from 23.05.2019) that the conducted research does not contradict the basic bioethical norms of the Council of Europe Convention on Human Rights and Biomedicine (1977), the Declaration of Helsinki, and also meet ethical and moral and legal requirements according to the order of the Ministry of Health of Ukraine №281 from 01.11.2000.

Odontometric study of the model of bone structures of the tooth-jaw complex in three projections was performed in the software shell i-Dixel One Volume Viewer (Ver.1.5.0, $\mathrm{J}$ Morita Mfg. Cor.). Measurements of the first and second molars of the right and left sides of the upper and lower jaws were performed: height of the tooth and crown of the tooth, length of the palatal medial and distal roots, vestibular-lingual and mesio-distal dimensions of the crown and neck of the tooth [18]. For convenience and compactness of the record, digital notation of teeth was used: the first digit indicates the quadrant number, and the second the ordinal number of the tooth, counting from the center line. Thus: 16 - upper right first molars; 17 - upper right second molars; 26 - upper left first molars; 27 - upper left second molars; 36 - lower left first molars; 37 - lower left second molars; 46 - lower right first molars; 47 - lower right second molars.

The cephalometric study involved measuring the parameters of the cerebral and facial parts of the head with a large Martin compass and a soft centimeter tape. Cephalometric measurements were performed in accordance with generally accepted recommendations and anatomical guidelines and points [1].

The shape of the head was determined by the ratio of the largest width of the head to the length of the head, and in accordance with the obtained value of the indicator made the distribution of craniotypes: up to 75.9 - dolichocephaly; from 76.0 to 80.9 - mesocephaly; from 81.0 to 85.4 brachycephaly; 85.5 and more - hyperbrachycephaly [23].

The type of face was calculated using the Garson index, according to which the morphological length of the face was divided by the largest width of the face, and according to the obtained value of the indicator made the distribution of face types: up to 78.9 - very wide face, 79.0-83.9 - wide face; 84.0-87.9 - average face; 88.0-92.9 - narrow face; 93.0 and more - a very narrow face [16].

The correlations between odontometric and cephalometric parameters were assessed using Spearman's nonparametric statistics in the licensed package "Statistica 6.1".

\section{Results}

The results of the correlation analysis between the sizes of molars of the upper and lower jaws with cephalometric indicators of the cerebral and facial skull of practically healthy men of the northern administrative-territorial region of Ukraine are presented in tables 1-6.

\section{Discussion}

The question of the relationship between the metric characteristics of cranial structures and odontometric parameters and the possibility of building models based on them, which can later find their practical application in dental practice, is being actively studied by scientists from different countries $[4,7,8,10]$. Such models can be used by physicians not only for an individual approach to each patient but also for screening mass examinations to predict the occurrence of a particular pathology, and thus create risk groups that will require special dental supervision in the future.

Recently, many studies have focused on the importance of ethnic and gender issues of cephalometric and odontometric indicators [5, 12, 13, 15, 17, 19-21], the results of which have a positive effect on improving dental health. Understanding the correlations of craniofacial structures allows the construction of mathematical models that can later find their practical application in dental practice $[4,7$, 
Polishchuk S.S., Kotsyura O.O., Dmitriev M.O., Orlovskiy V.O., Popov M.V.

Table 1. Correlations of the sizes of molars of an upper jaw with cephalometric indicators of a brain skull of men of the northern region of Ukraine $(n=10-32)$.

\begin{tabular}{|c|c|c|c|c|c|c|c|}
\hline \multirow{2}{*}{ Sizes of molars } & \multicolumn{7}{|c|}{ Cephalometric indicators } \\
\hline & DUG_GOP & DUGS_GOP & DUG_AUAU & G_OP & FMT_FMT & EY_EY & KRANIO \\
\hline $17 \mathrm{HZ}$ & 0.36 & 0.07 & 0.29 & 0.25 & 0.16 & 0.39 & 0.17 \\
\hline $17 \mathrm{HKZ}$ & 0.18 & 0.03 & 0.37 & 0.24 & 0.09 & $\underline{0.30}$ & 0.12 \\
\hline 17HRZ1 & 0.23 & 0.16 & 0.19 & 0.10 & 0.18 & $\underline{0.34}$ & 0.16 \\
\hline 17HRZ2 & 0.14 & 0.06 & 0.00 & 0.16 & 0.13 & -0.04 & -0.19 \\
\hline 17HRZ3 & 0.13 & -0.02 & 0.03 & -0.02 & 0.05 & 0.08 & 0.14 \\
\hline 17VO_K & 0.14 & 0.03 & 0.29 & -0.10 & 0.21 & 0.41 & 0.43 \\
\hline 17VO_S & 0.13 & 0.10 & 0.26 & -0.09 & 0.28 & 0.47 & 0.45 \\
\hline 17MD_K & 0.06 & 0.05 & 0.13 & 0.13 & 0.25 & 0.20 & 0.03 \\
\hline 17MD_S & 0.19 & 0.00 & 0.16 & 0.27 & 0.35 & 0.26 & -0.02 \\
\hline $16 \mathrm{HZ}$ & 0.02 & -0.04 & 0.20 & 0.00 & -0.15 & -0.03 & 0.05 \\
\hline $16 \mathrm{HKZ}$ & -0.02 & 0.23 & 0.17 & 0.16 & -0.21 & -0.03 & -0.16 \\
\hline 16HRZ1 & 0.06 & -0.21 & 0.10 & -0.14 & 0.09 & 0.01 & 0.14 \\
\hline 16HRZ2 & 0.07 & 0.10 & 0.28 & -0.11 & 0.16 & $\underline{0.31}$ & 0.36 \\
\hline $16 \mathrm{HRZ3}$ & 0.07 & -0.18 & 0.13 & -0.10 & -0.03 & -0.05 & 0.13 \\
\hline 16VO_S & 0.36 & 0.27 & 0.47 & 0.22 & 0.37 & 0.39 & 0.13 \\
\hline 16VO_K & 0.29 & 0.29 & $\underline{0.33}$ & 0.10 & 0.40 & 0.26 & 0.07 \\
\hline 16MD_S & 0.48 & 0.25 & $\underline{0.30}$ & 0.39 & 0.51 & 0.41 & 0.02 \\
\hline 16MD_K & 0.00 & -0.11 & 0.26 & -0.11 & 0.02 & 0.23 & $\underline{0.32}$ \\
\hline $26 \mathrm{HZ}$ & 0.03 & -0.12 & 0.16 & 0.03 & -0.02 & -0.14 & 0.00 \\
\hline $26 \mathrm{HKZ}$ & 0.16 & 0.21 & 0.20 & $\underline{0.34}$ & -0.04 & 0.00 & -0.24 \\
\hline 26HRZ1 & 0.00 & -0.16 & 0.02 & -0.03 & -0.06 & 0.10 & 0.20 \\
\hline 26HRZ2 & 0.04 & -0.05 & -0.06 & -0.12 & $\underline{0.32}$ & 0.08 & 0.13 \\
\hline $26 \mathrm{HRZ3}$ & -0.09 & -0.20 & 0.12 & -0.29 & -0.04 & -0.18 & 0.12 \\
\hline 26VO_K & 0.06 & 0.09 & 0.23 & -0.16 & 0.37 & 0.19 & 0.28 \\
\hline 26VO_S & 0.25 & 0.23 & 0.40 & 0.04 & $\underline{0.34}$ & 0.29 & 0.17 \\
\hline 26MD_K & 0.07 & -0.06 & $\underline{0.33}$ & 0.09 & -0.01 & 0.12 & 0.09 \\
\hline 26MD_S & 0.38 & $\underline{0.32}$ & 0.44 & $\underline{0.30}$ & 0.43 & 0.46 & 0.12 \\
\hline $27 \mathrm{HZ}$ & $\underline{0.32}$ & 0.04 & 0.28 & 0.09 & 0.29 & 0.54 & 0.39 \\
\hline $27 \mathrm{HKZ}$ & 0.21 & 0.13 & 0.44 & 0.29 & 0.03 & 0.22 & -0.04 \\
\hline 27HRZ1 & 0.10 & 0.10 & 0.14 & 0.04 & 0.29 & 0.15 & 0.06 \\
\hline $27 \mathrm{HRZ2}$ & 0.10 & 0.01 & 0.02 & 0.12 & 0.10 & 0.00 & -0.08 \\
\hline 27HRZ3 & 0.06 & -0.07 & 0.04 & -0.11 & 0.10 & 0.01 & 0.11 \\
\hline 27VO_K & 0.07 & -0.09 & 0.20 & -0.13 & 0.21 & 0.27 & 0.35 \\
\hline 27VO_S & 0.03 & -0.03 & 0.17 & -0.17 & 0.29 & $\underline{0.33}$ & 0.43 \\
\hline 27MD_K & 0.10 & 0.06 & 0.12 & 0.17 & $\underline{0.31}$ & 0.14 & -0.07 \\
\hline 27MD_S & 0.28 & 0.07 & 0.19 & $\underline{0.31}$ & 0.38 & $\underline{0.32}$ & -0.02 \\
\hline
\end{tabular}

Notes: here and in the following tables, bold and red highlighted - reliable medium-strength direct correlations; sand color and highlight - unreliable medium-strength direct correlations; HZ - tooth height; HKZ - height of the tooth crown; HRZ1 - length of the palatine root of the upper molars; HRZ2 - length of the dorsal proximal root of the upper molars; HRZ3 - length of the parietal distal root of the upper molars; VO K - vestibular-lingual size of the tooth crown; VO_S - vestibular-lingual size of the neck of the tooth; MD_K - mesio-distal size of the tooth crown; MD_S - mesio-distal size of the tooth neck; DUG GOP - the largest head circumference; DUGS GOP - sagittal arch; DUG AUAU - transverse arc; G OP - the largest length of the head; FMT_FMT - the smallest width of the head (frontal diameter); EU_EU - the largest width of the head (occipital diameter); KRANIO - cranial index. 
Correlations of linear sizes of molars with cephalometric indicators of practically healthy men of the northern...

Table 2. Correlations of the sizes of molars of a lower jaw with cephalometric indicators of a brain skull of men of the northern region of Ukraine $(n=10-32)$.

\begin{tabular}{|c|c|c|c|c|c|c|c|}
\hline \multirow{2}{*}{ Sizes of molars } & \multicolumn{7}{|c|}{ Cephalometric indicators } \\
\hline & DUG_GOP & DUGS_GOP & DUG_AUAU & G_OP & EY_EY & FMT_FMT & KRANIO \\
\hline $47 \mathrm{HZ}$ & 0.51 & 0.23 & 0.50 & 0.28 & 0.29 & 0.26 & -0.04 \\
\hline $47 \mathrm{HKZ}$ & 0.25 & 0.11 & 0.35 & 0.18 & 0.16 & 0.09 & -0.06 \\
\hline 47HRZ4 & 0.43 & 0.20 & 0.41 & 0.23 & 0.49 & 0.38 & 0.19 \\
\hline 47HRZ5 & 0.25 & 0.01 & 0.21 & 0.11 & 0.10 & 0.27 & -0.03 \\
\hline 47VO_K & 0.15 & 0.21 & 0.24 & 0.09 & 0.20 & 0.16 & 0.08 \\
\hline 47VO_S & -0.02 & 0.20 & 0.17 & -0.05 & 0.26 & 0.19 & $\underline{0.30}$ \\
\hline 47MD_K & 0.19 & 0.04 & 0.51 & 0.12 & $\underline{0.31}$ & 0.18 & 0.17 \\
\hline 47MD_S & 0.23 & 0.08 & 0.36 & 0.07 & 0.40 & 0.49 & 0.24 \\
\hline $46 \mathrm{HZ}$ & $\underline{0.32}$ & $\underline{0.35}$ & $\underline{0.32}$ & 0.37 & 0.28 & 0.29 & -0.06 \\
\hline $46 \mathrm{HKZ}$ & 0.43 & 0.39 & 0.46 & $\underline{0.32}$ & 0.40 & $\underline{0.30}$ & 0.07 \\
\hline $46 \mathrm{HRZ4}$ & 0.13 & 0.02 & 0.00 & 0.28 & -0.06 & 0.00 & -0.27 \\
\hline 46HRZ5 & 0.29 & 0.23 & 0.18 & $\underline{0.34}$ & 0.26 & 0.38 & -0.04 \\
\hline 46VO_K & -0.12 & 0.01 & 0.25 & 0.11 & 0.02 & -0.05 & -0.05 \\
\hline 46VO_S & -0.16 & 0.03 & 0.06 & -0.02 & 0.10 & 0.10 & 0.15 \\
\hline 46MD_K & 0.10 & 0.01 & 0.21 & 0.27 & 0.09 & 0.15 & -0.11 \\
\hline 46MD_S & 0.29 & 0.23 & 0.39 & 0.41 & 0.27 & 0.29 & -0.13 \\
\hline $36 \mathrm{HZ}$ & 0.23 & 0.24 & 0.11 & 0.26 & 0.06 & 0.16 & -0.16 \\
\hline $36 \mathrm{HKZ}$ & $\underline{0.31}$ & $\underline{0.30}$ & 0.36 & 0.25 & 0.23 & 0.01 & 0.02 \\
\hline $36 \mathrm{HRZ4}$ & 0.08 & 0.08 & -0.08 & 0.14 & -0.12 & 0.13 & -0.25 \\
\hline $36 \mathrm{HRZ5}$ & 0.14 & 0.20 & 0.03 & 0.18 & 0.11 & 0.49 & -0.05 \\
\hline 36VO_K & -0.12 & 0.01 & 0.20 & 0.14 & -0.07 & 0.05 & -0.14 \\
\hline 36VO_S & -0.08 & 0.10 & 0.14 & 0.02 & 0.15 & 0.20 & 0.16 \\
\hline 36MD_K & 0.06 & 0.09 & 0.29 & $\underline{0.32}$ & 0.07 & 0.15 & -0.18 \\
\hline 36MD_S & 0.35 & 0.26 & 0.38 & 0.43 & 0.19 & 0.39 & -0.21 \\
\hline $37 \mathrm{HZ}$ & 0.49 & 0.18 & 0.35 & $\underline{0.34}$ & $\underline{0.32}$ & 0.24 & -0.02 \\
\hline $37 \mathrm{HKZ}$ & 0.29 & 0.16 & 0.36 & 0.27 & 0.23 & 0.12 & -0.05 \\
\hline 37HRZ4 & 0.38 & 0.05 & 0.17 & 0.18 & $\underline{0.33}$ & $\underline{0.32}$ & 0.12 \\
\hline 37HRZ5 & 0.27 & -0.02 & 0.13 & 0.18 & 0.18 & $\underline{0.30}$ & 0.04 \\
\hline 37VO_K & -0.05 & 0.11 & 0.20 & -0.03 & 0.09 & 0.12 & 0.12 \\
\hline 37VO_S & -0.14 & 0.01 & 0.05 & -0.19 & 0.15 & 0.16 & $\underline{0.34}$ \\
\hline 37MD_K & $\underline{0.31}$ & 0.13 & 0.53 & 0.25 & $\underline{0.33}$ & 0.21 & 0.09 \\
\hline 37MD_s & 0.42 & 0.12 & 0.48 & 0.19 & 0.57 & 0.48 & $\underline{0.32}$ \\
\hline
\end{tabular}

Notes: here and in the following tables, HRZ4 - the length of the near root of the lower molars; HRZ5 - the length of the distal root of the lower molars.

Table 3. Correlations of the sizes of molars of an upper jaw with cephalometric indicators of a face skull of men of the northern region of Ukraine ( $n=10-32)$.

\begin{tabular}{|c|c|c|c|c|c|c|c|c|c|}
\hline \multirow{2}{*}{ Sizes of molars } & \multicolumn{10}{|c|}{ Cephalometric indicators } \\
\cline { 2 - 22 } & ZY_ZY & ZM_ZM & TR_GN & TR_N & N_GN & N_PRN & N_SN & GO_GO & N_STO \\
\hline $17 \mathrm{HZ}$ & 0.08 & 0.12 & 0.22 & 0.14 & 0.05 & $\underline{0.32}$ & $\underline{0.34}$ & 0.18 & 0.20 \\
\hline $17 \mathrm{HKZ}$ & 0.15 & -0.20 & -0.01 & 0.27 & -0.24 & $\underline{0.30}$ & 0.37 & 0.18 & 0.28 \\
\hline $17 \mathrm{HRZ1}$ & 0.22 & 0.14 & 0.03 & 0.05 & -0.14 & 0.24 & 0.20 & 0.05 & 0.00 \\
\hline
\end{tabular}


Polishchuk S.S., Kotsyura O.O., Dmitriev M.O., Orlovskiy V.O., Popov M.V.

Table 3. (continuation)

\begin{tabular}{|c|c|c|c|c|c|c|c|c|c|}
\hline \multirow{2}{*}{ Sizes of molars } & \multicolumn{9}{|c|}{ Cephalometric indicators } \\
\hline & ZY_ZY & ZM_ZM & TR_GN & TR_N & N_GN & N_PRN & N_SN & GO_GO & N_STO \\
\hline 17HRZ2 & -0.14 & 0.18 & 0.21 & -0.07 & 0.24 & 0.07 & 0.10 & 0.15 & -0.09 \\
\hline 17HRZ3 & 0.15 & $\underline{0.37}$ & 0.07 & -0.01 & 0.21 & 0.27 & 0.17 & 0.19 & 0.13 \\
\hline 17VO_K & 0.05 & 0.03 & 0.01 & -0.07 & -0.06 & 0.07 & 0.08 & 0.19 & -0.08 \\
\hline 17VO_S & 0.10 & -0.02 & 0.01 & -0.04 & -0.06 & 0.00 & 0.00 & 0.14 & -0.15 \\
\hline 17MD_K & 0.20 & -0.16 & -0.05 & -0.02 & -0.15 & 0.08 & 0.10 & 0.25 & -0.12 \\
\hline 17MD_S & 0.17 & -0.01 & -0.10 & 0.01 & -0.10 & 0.16 & 0.24 & 0.08 & -0.14 \\
\hline $16 \mathrm{HZ}$ & 0.01 & -0.02 & -0.04 & -0.10 & -0.10 & 0.22 & 0.25 & 0.16 & 0.15 \\
\hline $16 \mathrm{HKZ}$ & 0.11 & $\underline{-0.30}$ & -0.10 & -0.05 & -0.17 & 0.15 & 0.22 & 0.10 & 0.29 \\
\hline 16HRZ1 & -0.05 & 0.21 & -0.02 & -0.11 & -0.09 & 0.10 & 0.08 & -0.07 & -0.06 \\
\hline 16HRZ2 & 0.11 & 0.04 & -0.27 & -0.17 & -0.18 & -0.02 & -0.04 & 0.17 & 0.05 \\
\hline $16 \mathrm{HRZ3}$ & -0.05 & 0.27 & 0.22 & -0.01 & 0.06 & 0.18 & 0.12 & 0.11 & 0.06 \\
\hline 16VO_S & 0.19 & -0.08 & 0.04 & 0.05 & -0.05 & 0.14 & 0.19 & 0.17 & -0.11 \\
\hline 16VO_K & 0.08 & -0.10 & 0.20 & 0.12 & -0.04 & -0.02 & 0.02 & $\underline{0.30}$ & -0.06 \\
\hline 16MD_S & 0.18 & 0.41 & 0.19 & 0.08 & 0.41 & -0.15 & -0.03 & 0.02 & -0.26 \\
\hline 16MD_K & -0.01 & 0.11 & 0.02 & -0.03 & -0.04 & -0.08 & -0.09 & -0.06 & 0.23 \\
\hline $26 \mathrm{HZ}$ & 0.05 & 0.06 & 0.00 & -0.11 & 0.09 & 0.27 & 0.26 & 0.20 & $\underline{0.35}$ \\
\hline $26 \mathrm{HKZ}$ & 0.12 & -0.16 & -0.02 & 0.00 & 0.01 & 0.16 & 0.21 & 0.15 & 0.35 \\
\hline $26 \mathrm{HRZ1}$ & -0.05 & 0,15 & -0.11 & 0.08 & -0.15 & 0.24 & 0.19 & -0.05 & 0.11 \\
\hline 26HRZ2 & -0.04 & 0.37 & -0.10 & -0.18 & 0.06 & -0.04 & -0.09 & 0.26 & -0.20 \\
\hline $26 \mathrm{HRZ3}$ & 0.00 & 0.20 & 0.18 & -0.12 & 0.13 & 0.06 & 0.04 & 0.17 & 0.19 \\
\hline 26VO_K & 0.09 & -0.08 & 0.22 & 0.13 & -0.08 & 0.08 & 0.07 & $\underline{0.34}$ & -0.03 \\
\hline 26VO_S & 0.14 & -0.06 & 0.17 & 0.19 & -0.02 & 0.06 & 0.06 & $\underline{0.30}$ & 0.03 \\
\hline 26MD_K & -0.04 & 0.13 & -0.02 & -0.09 & -0.02 & -0.02 & 0.00 & -0.05 & 0.20 \\
\hline 26MD_S & 0.27 & 0.24 & 0.16 & 0.11 & $\underline{0.33}$ & -0.17 & -0.07 & 0.10 & -0.21 \\
\hline $27 \mathrm{HZ}$ & 0.13 & 0.30 & 0.13 & -0.02 & 0.10 & 0.00 & -0.02 & 0.06 & 0.06 \\
\hline $27 \mathrm{HKZ}$ & 0.10 & -0.24 & -0.06 & 0.07 & -0.25 & 0.16 & 0.20 & 0.02 & 0.27 \\
\hline 27HRZ1 & 0.24 & 0.21 & 0.00 & -0.07 & -0.12 & 0.21 & 0.15 & 0.12 & -0.04 \\
\hline 27HRZ2 & -0.03 & 0.22 & 0.14 & -0.19 & $\underline{0.30}$ & -0.04 & -0.08 & 0.05 & 0.03 \\
\hline 27HRZ3 & 0.04 & 0.44 & 0.08 & -0.11 & 0.12 & 0.06 & 0.04 & 0.11 & 0.04 \\
\hline 27VO_K & -0.10 & 0.11 & 0.09 & -0.08 & -0.06 & -0.07 & -0.07 & 0.08 & 0.00 \\
\hline 27VO_S & -0.03 & 0.09 & 0.15 & 0.01 & -0.02 & -0.07 & -0.07 & 0.09 & -0.02 \\
\hline 27MD_K & 0.11 & -0.11 & 0.05 & 0.04 & -0.03 & 0.01 & 0.02 & 0.22 & -0.09 \\
\hline 27MD_s & 0.23 & 0.04 & -0.02 & -0.03 & 0.05 & 0.20 & 0.24 & 0.05 & -0.17 \\
\hline
\end{tabular}

Notes: here and in the following tables, inaccurate mean correlations of inverse correlations are highlighted by underlining and green color; $Z Y$ ZY - face width (distance between zygomatic points); ZM_ZM - average width of the face; TR_GN - physiological length of the face; TR_N - forehead height; N_GN - morphological length of the face; N_PRN - length of the nose; N_SN - nose height; GO_GO width of the lower jaw (width between the corners of the lower jaw); N_STO - height of the upper part of the face.

Table 4. Correlations of the sizes of molars of a lower jaw with cephalometric indicators of a face skull of men of the northern region of Ukraine $(n=10-32)$.

\begin{tabular}{|c|c|c|c|c|c|c|c|c|c|}
\hline \multirow{2}{*}{ Sizes of molars } & \multicolumn{10}{|c|}{ Cephalometric indicators } \\
\cline { 2 - 11 } & ZY_ZY & ZM_ZM & TR_GN & TR_N & N_GN & N_PRN & N_SN & GO_GO & N_STO \\
\hline $47 \mathrm{HZ}$ & 0.15 & 0.10 & 0.20 & 0.04 & 0.09 & 0.13 & 0.14 & 0.12 & 0.07 \\
\hline $47 \mathrm{HKZ}$ & -0.03 & -0.01 & -0.08 & -0.07 & -0.10 & 0.04 & 0.10 & -0.10 & -0.07 \\
\hline
\end{tabular}


Correlations of linear sizes of molars with cephalometric indicators of practically healthy men of the northern...

Table 4. (continuation)

\begin{tabular}{|c|c|c|c|c|c|c|c|c|c|}
\hline \multirow{2}{*}{ Sizes of molars } & \multicolumn{9}{|c|}{ Cephalometric indicators } \\
\hline & ZY_ZY & ZM_ZM & TR_GN & TR_N & N_GN & N_PRN & N_SN & GO_GO & N_STO \\
\hline 47HRZ4 & 0.18 & 0.10 & 0.15 & 0.13 & 0.12 & 0.05 & 0.03 & 0.13 & -0.01 \\
\hline 47HRZ5 & 0.14 & 0.01 & 0.16 & 0.00 & 0.23 & 0.07 & 0.02 & 0.27 & 0.00 \\
\hline 47VO_K & 0.04 & -0.16 & 0.15 & -0.06 & -0.02 & 0.04 & 0.00 & 0.26 & -0.01 \\
\hline 47VO_S & 0.08 & -0.16 & -0.01 & 0.06 & -0.09 & 0.09 & 0.06 & 0.22 & -0.04 \\
\hline 47MD_K & -0.04 & -0.01 & 0.03 & 0.13 & -0.13 & 0.09 & 0.10 & 0.08 & 0.17 \\
\hline 47MD_S & 0.11 & 0.05 & -0.11 & 0.05 & -0.04 & 0.03 & 0.02 & 0.18 & -0.24 \\
\hline $46 \mathrm{HZ}$ & $\underline{0.34}$ & 0.19 & 0.15 & $\underline{0.31}$ & 0.03 & 0.16 & 0.16 & $\underline{0.30}$ & -0.07 \\
\hline $46 \mathrm{HKZ}$ & 0.38 & 0.07 & 0.26 & 0.43 & 0.08 & 0.29 & 0.35 & 0.36 & 0.25 \\
\hline 46HRZ4 & -0.02 & 0.27 & -0.11 & 0.05 & -0.18 & 0.03 & 0.04 & -0.14 & -0.05 \\
\hline $46 \mathrm{HRZ5}$ & 0.18 & 0.27 & 0.18 & $\underline{0.31}$ & 0.00 & -0.06 & -0.01 & 0.20 & 0.00 \\
\hline 46VO_K & 0.03 & -0.40 & 0.07 & 0.10 & -0.20 & 0.22 & 0.20 & $\underline{0.31}$ & 0.16 \\
\hline 46VO_S & 0.05 & -0.21 & -0.08 & 0.13 & -0.29 & 0.15 & 0.15 & 0.25 & 0.03 \\
\hline 46MD_K & -0.14 & 0.03 & 0.01 & 0.09 & -0.04 & 0.06 & 0.12 & 0.11 & 0.08 \\
\hline 46MD_S & 0.21 & -0.01 & 0.02 & 0.08 & -0.15 & 0.20 & 0.23 & -0.10 & -0.03 \\
\hline $36 \mathrm{HZ}$ & 0.18 & 0.21 & 0.24 & $\underline{0.35}$ & 0.09 & 0.06 & 0.11 & 0.20 & 0.09 \\
\hline $36 \mathrm{HKZ}$ & 0.29 & -0.08 & 0.24 & 0.37 & 0.06 & 0.26 & $\underline{0.31}$ & 0.15 & 0.44 \\
\hline $36 \mathrm{HRZ4}$ & -0.03 & 0.35 & -0.03 & 0.00 & -0.06 & -0.13 & -0.11 & -0.04 & -0.16 \\
\hline $36 \mathrm{HRZ5}$ & 0.24 & 0.28 & 0.26 & 0.35 & -0.03 & -0.02 & 0.06 & 0.22 & 0.01 \\
\hline 36VO_K & 0.01 & -0.28 & 0.18 & 0.21 & -0.11 & 0.17 & 0.14 & $\underline{0.31}$ & 0.15 \\
\hline 36VO_S & 0.11 & -0.13 & -0.07 & 0.07 & -0.24 & 0.03 & -0.01 & $\underline{0.31}$ & -0.06 \\
\hline 36MD_K & -0.10 & -0.10 & -0.01 & 0.06 & -0.07 & 0.05 & 0.08 & 0.10 & -0.07 \\
\hline 36MD_S & 0.11 & 0.13 & 0.23 & 0.13 & 0.09 & 0.05 & 0.06 & 0.07 & -0.07 \\
\hline $37 \mathrm{HZ}$ & 0.12 & 0.16 & 0.17 & 0.12 & 0.07 & 0.17 & 0.19 & 0.04 & 0.19 \\
\hline $37 \mathrm{HKZ}$ & 0.08 & 0.00 & 0.01 & 0.11 & -0.11 & 0.16 & 0.23 & -0.11 & 0.07 \\
\hline 37HRZ4 & 0.02 & 0.25 & 0.08 & -0.01 & 0.11 & -0.03 & -0.02 & -0.05 & 0.05 \\
\hline 37HRZ5 & 0.12 & 0.20 & 0.13 & -0.06 & 0.26 & 0.00 & 0.00 & 0.14 & -0.05 \\
\hline 37VO_K & 0.07 & -0.27 & 0.14 & 0.03 & -0.11 & 0.09 & 0.07 & $\underline{0.34}$ & 0.14 \\
\hline 37VO_S & 0.02 & -0.08 & -0.10 & -0.05 & -0.10 & -0.06 & -0.11 & $\underline{0.31}$ & -0.06 \\
\hline 37MD_K & -0.03 & 0.06 & 0.13 & 0.17 & -0.02 & 0.15 & 0.14 & 0.03 & 0.08 \\
\hline 37MD_S & 0.09 & 0.13 & 0.00 & 0.06 & 0.06 & 0.08 & 0.11 & 0.05 & -0.18 \\
\hline
\end{tabular}

Note: here and in the following tables, bold and blue highlighted significant medium-strength inverse correlations.

Table 5. Correlations of the sizes of molars of an upper jaw with cephalometric indicators of a face skull of men of the northern region of Ukraine $(n=10-32)$.

\begin{tabular}{|c|c|c|c|c|c|c|c|c|c|}
\hline \multirow{2}{*}{ Sizes of molars } & \multicolumn{10}{|c|}{ Cephalometric indicators } \\
\cline { 2 - 12 } & SN_PRN & AL_AL & CH__CHI & EK_EK & MF_MF & N_I & PGO_GN & IGO_GN & IN_GARS \\
\hline $17 \mathrm{HZ}$ & -0.01 & 0.04 & -0.09 & $\underline{0.32}$ & 0.17 & -0.03 & 0.20 & 0.25 & -0.08 \\
\hline $17 \mathrm{HKZ}$ & 0.06 & -0.20 & -0.14 & 0.29 & 0.02 & -0.12 & 0.16 & 0.27 & -0.24 \\
\hline $17 \mathrm{HRZ1}$ & 0.17 & 0.28 & 0.19 & 0.28 & 0.07 & -0.04 & 0.28 & $\underline{0.32}$ & -0.31 \\
\hline $17 \mathrm{HRZ2}$ & -0.12 & -0.02 & 0.00 & -0.02 & 0.15 & -0.06 & -0.10 & -0.19 & 0.12 \\
\hline $17 \mathrm{HRZ3}$ & -0.08 & -0.07 & 0.14 & -0.06 & 0.19 & -0.04 & 0.13 & 0.12 & -0.03 \\
\hline $17 \mathrm{VO} K$ & -0.31 & 0.11 & 0.01 & 0.36 & 0.10 & -0.01 & -0.03 & 0.07 & -0.21 \\
\hline
\end{tabular}


Polishchuk S.S., Kotsyura O.O., Dmitriev M.O., Orlovskiy V.O., Popov M.V.

Table 5. (continuation)

\begin{tabular}{|c|c|c|c|c|c|c|c|c|c|}
\hline \multirow{2}{*}{ Sizes of molars } & \multicolumn{9}{|c|}{ Cephalometric indicators } \\
\hline & SN_PRN & AL_AL & CHI_CHI & EK_EK & MF_MF & N_l & PGO_GN & IGO_GN & IN_GARS \\
\hline 17VO_S & -0.30 & 0.14 & 0.01 & $\underline{0.33}$ & 0.15 & -0.07 & 0.00 & 0.02 & 0.18 \\
\hline 17MD_K & -0.11 & -0.16 & -0.12 & 0.35 & -0.11 & 0.04 & 0.02 & 0.17 & $\underline{-0.30}$ \\
\hline 17MD_S & -0.14 & -0.02 & -0.19 & 0.25 & -0.05 & -0.10 & 0.03 & 0.20 & -0.28 \\
\hline $16 \mathrm{HZ}$ & -0.02 & -0.04 & 0.07 & 0.24 & 0.10 & 0.09 & -0.04 & 0.03 & -0.04 \\
\hline $16 \mathrm{HKZ}$ & 0.03 & -0.15 & -0.17 & 0.19 & -0.05 & -0.17 & 0.14 & 0.10 & -0.07 \\
\hline 16HRZ1 & 0.12 & -0.01 & 0.36 & 0.19 & 0.06 & 0.02 & -0.13 & -0.05 & 0.00 \\
\hline 16HRZ2 & -0.11 & -0.02 & 0.00 & 0.21 & -0.09 & 0.09 & 0.10 & 0.09 & -0.23 \\
\hline 16HRZ3 & -0.03 & -0.08 & 0.15 & 0.08 & 0.18 & 0.20 & -0.10 & 0.08 & 0.10 \\
\hline 16VO_S & -0.25 & $\underline{-0.32}$ & -0.41 & 0.21 & -0.06 & -0.01 & 0.01 & 0.22 & -0.15 \\
\hline 16VO_K & -0.06 & -0.10 & -0.19 & 0.16 & -0.11 & 0.17 & 0.00 & 0.09 & -0.16 \\
\hline 16MD_S & -0.06 & -0.14 & -0.28 & 0.18 & 0.21 & 0.15 & 0.09 & 0.19 & -0.06 \\
\hline 16MD_K & 0.20 & -0.22 & 0.02 & 0.17 & 0.03 & 0.05 & -0.06 & 0.05 & -0.03 \\
\hline $26 \mathrm{HZ}$ & 0.05 & $\underline{-0.32}$ & -0.14 & 0.12 & 0.01 & 0.29 & -0.05 & 0.02 & 0.18 \\
\hline $26 \mathrm{HKZ}$ & 0.03 & -0.21 & -0.21 & 0.22 & -0.03 & 0.03 & 0.20 & 0.18 & 0.04 \\
\hline $26 \mathrm{HRZ1}$ & 0.02 & -0.15 & 0.07 & -0.01 & 0.08 & -0.10 & 0.07 & 0.13 & -0.08 \\
\hline 26HRZ2 & -0.06 & -0.02 & -0.04 & -0.05 & -0.24 & 0.14 & -0.05 & 0.06 & -0.09 \\
\hline $26 \mathrm{HRZ3}$ & 0,18 & -0.07 & 0.21 & 0.04 & 0.16 & 0.16 & -0.21 & -0.13 & 0.18 \\
\hline 26VO_K & -0.09 & -0.14 & -0.12 & 0.17 & -0.10 & 0.08 & -0.16 & -0.01 & -0.20 \\
\hline 26VO_S & 0.01 & -0.27 & $\underline{-0.33}$ & -0.04 & -0.05 & -0.01 & -0.05 & 0.09 & -0.15 \\
\hline 26MD_K & 0.09 & $\underline{-0.31}$ & -0.07 & $\underline{0.32}$ & 0.02 & 0.17 & -0.02 & 0.16 & 0.15 \\
\hline 26MD_S & -0.03 & -0.10 & -0.15 & 0.16 & $\underline{0.30}$ & 0.02 & 0.06 & 0.11 & -0.19 \\
\hline $27 \mathrm{HZ}$ & 0.01 & 0.16 & 0.21 & 0.35 & 0.25 & 0.06 & 0.13 & 0.16 & -0.24 \\
\hline $27 \mathrm{HKZ}$ & 0.12 & -0.29 & -0.22 & 0.22 & 0.00 & -0.14 & 0.14 & 0.25 & -0.13 \\
\hline 27HRZ1 & 0.09 & 0.10 & 0.20 & 0.27 & -0.01 & 0.05 & 0.13 & 0.21 & -0.25 \\
\hline 27HRZ2 & -0.07 & -0.05 & 0.15 & 0.01 & 0.24 & 0.09 & -0.06 & -0.22 & 0.08 \\
\hline $27 \mathrm{HRZ3}$ & -0.05 & 0.08 & $\underline{0.34}$ & 0.06 & 0.21 & -0.01 & -0.05 & -0.13 & -0.09 \\
\hline 27VO_K & -0.23 & 0.17 & 0.14 & 0.37 & 0.13 & 0.17 & -0.15 & -0.13 & $-0,14$ \\
\hline 27VO_S & -0.20 & 0.15 & 0.13 & $\underline{0.33}$ & 0.13 & 0.13 & -0.15 & -0.17 & -0.21 \\
\hline 27MD_K & 0,00 & -0.25 & -0.21 & 0.15 & -0.12 & 0.05 & -0.03 & 0.10 & -0.19 \\
\hline 27MD_S & -0.11 & -0.05 & -0.25 & 0.16 & -0.01 & -0.09 & 0.08 & 0.26 & -0.22 \\
\hline
\end{tabular}

Notes: here and in the following tables, SN_PRN -the depth of the nose; AL_AL - width of the base of the nose (distance between alar points); CHI_CHI - width of the oral slit; EK_EK - extraocular width (biorbital width); MF_MF - interorbital width (anterior interorbital width); N_I - the distance between the nasion and the intercanine point; RGO_GN - body length of the lower jaw on the right; LGO_GN - body length of the lower jaw on the left; IN_GARS - Garson's morphological index.

Table 6. Correlations of the sizes of molars of a lower jaw with cephalometric indicators of a face skull of men of the northern region of Ukraine $(n=10-32)$.

\begin{tabular}{|c|c|c|c|c|c|c|c|c|c|}
\hline \multirow{2}{*}{ Sizes of molars } & \multicolumn{9}{|c|}{ Cephalometric indicators } \\
\hline & SN_PRN & $A L \_A L$ & $\mathrm{CHI}$ & EK_EK & MF_MF & N_l & PGO_GN & IGO_GN & IN_GARS \\
\hline $47 \mathrm{HZ}$ & 0.24 & -0.02 & 0.07 & 0.14 & 0.19 & 0.07 & 0.01 & 0.19 & -0.06 \\
\hline $47 \mathrm{HKZ}$ & 0.12 & -0.18 & -0.16 & 0.24 & 0.05 & -0.22 & -0.05 & 0.06 & -0.08 \\
\hline 47HRZ4 & -0.06 & 0.25 & 0.23 & 0.16 & 0.27 & 0.01 & 0.14 & 0.22 & -0.17 \\
\hline 47HRZ5 & 0.26 & -0.15 & 0.00 & -0.04 & -0.01 & 0.28 & -0.17 & 0.10 & 0.10 \\
\hline 47VO_K & -0.33 & 0.16 & 0.01 & 0.17 & 0.08 & 0.10 & 0.08 & 0.12 & -0.10 \\
\hline
\end{tabular}


Correlations of linear sizes of molars with cephalometric indicators of practically healthy men of the northern...

Table 6. (continuation)

\begin{tabular}{|c|c|c|c|c|c|c|c|c|c|}
\hline \multirow{2}{*}{ Sizes of molars } & \multicolumn{9}{|c|}{ Cephalometric indicators } \\
\hline & SN_PRN & AL_AL & $\mathrm{CHI}$ & EK_EK & MF_MF & N_l & PGO_GN & IGO_GN & IN_GARS \\
\hline 47VO_S & $\underline{-0.34}$ & 0.03 & -0.23 & -0.03 & 0.03 & -0.01 & 0.08 & 0.04 & -0.17 \\
\hline 47MD_K & -0.20 & -0.18 & 0.03 & 0.35 & 0.13 & -0.08 & -0.10 & 0.07 & -0.09 \\
\hline 47MD_S & -0.26 & -0.04 & -0.07 & 0.11 & 0.03 & -0.17 & -0.04 & 0.08 & -0.24 \\
\hline $46 \mathrm{HZ}$ & 0.15 & -0.23 & -0.18 & 0.05 & 0.08 & 0.01 & 0.23 & 0.51 & -0.29 \\
\hline $46 \mathrm{HKZ}$ & 0.21 & -0.20 & -0.17 & 0.25 & 0.07 & -0.01 & 0.24 & 0.51 & -0.19 \\
\hline 46HRZ4 & 0.13 & 0.04 & 0.09 & -0.02 & 0.07 & 0.03 & 0.06 & 0.27 & -0.07 \\
\hline $46 \mathrm{HRZ} 5$ & 0.16 & -0.09 & -0.12 & 0.11 & -0.04 & 0.29 & 0.26 & 0.44 & -0.21 \\
\hline 46VO_K & -0.14 & -0.27 & -0.11 & 0.26 & -0.01 & -0.04 & -0.16 & -0.02 & -0.08 \\
\hline 46VO_S & -0.26 & -0.08 & -0.13 & 0.12 & -0.12 & 0.10 & -0.06 & -0.02 & -0.25 \\
\hline 46MD_K & -0.24 & -0.19 & -0.16 & 0.25 & 0.04 & 0.01 & -0.19 & 0.05 & 0.13 \\
\hline 46MD_S & -0.15 & -0.29 & -0.16 & 0.23 & 0.16 & -0.09 & -0.08 & 0.14 & $\underline{-0.35}$ \\
\hline $36 \mathrm{HZ}$ & 0.44 & -0.15 & -0.14 & -0.01 & 0.01 & 0.13 & 0.15 & $\underline{0.31}$ & -0.05 \\
\hline $36 \mathrm{HKZ}$ & 0.35 & -0.21 & -0.19 & 0.13 & 0.12 & -0.01 & 0.23 & $\underline{0.31}$ & -0.07 \\
\hline 36HRZ4 & 0.17 & 0.01 & 0.10 & -0.12 & -0.02 & 0.07 & -0.05 & 0.14 & -0.03 \\
\hline $36 \mathrm{HRZ5}$ & 0.18 & -0.24 & -0.18 & 0.08 & -0.10 & 0.20 & 0.13 & 0.25 & -0.27 \\
\hline 36VO_K & -0.10 & -0.28 & -0.10 & 0.18 & 0.03 & 0.03 & -0.18 & -0.04 & -0.03 \\
\hline 36VO_S & $\underline{-0.33}$ & -0.16 & -0.20 & 0.09 & -0.09 & 0.09 & -0.01 & 0.07 & -0.27 \\
\hline 36MD_K & -0.38 & -0.28 & -0.23 & 0.23 & 0.08 & -0.12 & -0.18 & 0.06 & 0.04 \\
\hline 36MD_S & -0.15 & -0.25 & -0.17 & 0.11 & $\underline{0.30}$ & 0.04 & -0.14 & 0.02 & -0.17 \\
\hline $37 \mathrm{HZ}$ & 0.24 & 0.01 & -0.04 & 0.12 & 0.18 & 0.08 & 0.09 & 0.21 & -0.10 \\
\hline $37 \mathrm{HKZ}$ & 0.27 & -0.20 & -0.20 & 0.24 & 0.05 & -0.16 & 0.05 & 0.16 & -0.16 \\
\hline 37HRZ4 & -0.04 & 0.28 & 0.21 & 0.17 & 0.16 & 0.12 & 0.07 & 0.14 & -0.04 \\
\hline 37HRZ5 & 0.19 & -0.19 & -0.14 & 0.08 & 0.05 & 0.24 & -0.11 & 0.13 & 0.08 \\
\hline 37VO_K & -0.28 & 0.06 & 0.04 & 0.18 & -0.01 & 0.13 & 0.00 & 0.00 & -0.13 \\
\hline 37VO_S & -0.43 & 0.15 & 0.01 & 0.00 & -0.01 & 0.09 & 0.02 & -0.05 & -0.17 \\
\hline 37MD_K & -0.28 & -0.09 & 0.08 & 0.38 & 0.23 & -0.06 & -0.01 & 0.21 & -0.03 \\
\hline 37MD_S & $\underline{-0.30}$ & -0.08 & -0.13 & $\underline{0.30}$ & 0.16 & -0.14 & -0.02 & 0.17 & -0.16 \\
\hline
\end{tabular}

$8,10]$ and in forensic medicine [2] because such models can be used by doctors to create an individual diagnostic approach to each patient.

In the analysis of reliable and average strength of unreliable correlations of CT sizes of molars with cephalometric indicators and indices of practically healthy men from the northern region of Ukraine, the multiple nature of direct reliable $(r=0.35-0.57)$ and average strength of unreliable $(r=0.30-0.35)$ correlations was established only with indicators of a brain skull.

Our quantitative analysis of reliable and average strength of unreliable correlations of CT sizes of molars with cephalometric indicators and indices of practically healthy men of the northern region of Ukraine revealed the following distribution of correlations:

between the upper molars and indicators of a brain skull 47 correlations from 252 possible (18.7\%), from which,
$11.9 \%$ of direct reliable average force and $6.7 \%$ of direct unreliable average force, among which - with the first teeth 26 correlations from 126 possible $(12.7 \%$ of direct reliable medium strength and $7.9 \%$ of direct unreliable medium strength); with second teeth 21 correlations from 126 possible $(11.1 \%$ of direct reliable average forces and $5.6 \%$ of direct unreliable average forces); with the height of the teeth, their crowns and the length of the roots 13 correlations out of 140 possible $(5.0 \%$ of direct reliable medium strength and $4.3 \%$ of direct unreliable medium strength); with vestibular-lingual and mesio-distal dimensions 34 correlations out of 112 possible $(20.5 \%$ of direct reliable average forces and $9.8 \%$ of direct unreliable average forces);

between the lower molars and indicators of a brain skull 54 correlations from 224 possible $(24.1 \%)$, from which, $15.2 \%$ of direct reliable average force and $8.9 \%$ of direct 
unreliable average force, among which - with the first teeth 23 correlations from 112 possible $(12.5 \%$ of direct reliable medium strength and $8.0 \%$ of direct unreliable medium strength); with second teeth 31 correlations from 112 possible (17.9\% of direct reliable average forces and $9.8 \%$ of direct unreliable average forces); with the height of the teeth, their crowns and the length of the roots 32 correlations out of 112 possible $(20.0 \%$ of direct reliable medium strength and $11.6 \%$ of direct unreliable medium strength); with vestibular-lingual and mesio-distal dimensions 22 correlations out of 112 possible $(13.4 \%$ of direct reliable average forces and $6.2 \%$ of direct unreliable average forces);

between the upper molars and indicators of the facial skull 39 correlations out of 648 possible $(6.0 \%)$, of which, $1.7 \%$ of direct reliable medium force, $2.8 \%$ of direct unreliable medium force, $0.2 \%$ of reverse reliable medium force and $1.4 \%$ of inverse unreliable medium force, among which - with the first teeth 18 correlations out of 324 possible ( $1.5 \%$ of direct reliable of medium strength, $2.2 \%$ of direct unreliable of medium strength, $0.3 \%$ of inverse reliable of medium strength and $1.5 \%$ of inverse unreliable of medium strength); with second teeth 21 correlations from 324 possible $(1.9 \%$ of direct reliable average forces, $3.4 \%$ of direct unreliable average forces and $1.2 \%$ of inverse unreliable average forces); with the height of the teeth, their crowns and the length of the roots, 19 correlations out of 360 possible $(1.7 \%$ of direct reliable medium strength, $2.8 \%$ of direct unreliable medium strength and $0.8 \%$ of inverse unreliable medium strength); with vestibular-lingual and mesio-distal dimensions 20 correlations out of 288 possible $(1.7 \%$ of direct reliable medium forces, $2.8 \%$ of direct unreliable medium forces, $0.3 \%$ of reverse reliable medium forces and $2.1 \%$ of inverse unreliable medium forces);

between lower molars and indicators of a facial skull 38 correlations from 576 possible $(6.6 \%)$, from which, $2.6 \%$ of direct reliable average force, $2.6 \%$ of direct unreliable average force, $0.5 \%$ of return reliable average force and $0.9 \%$ of return unreliable average force, among which with the first teeth 29 correlations out of 288 possible $(4.5 \%$

\section{References}

[1] Bunak, V. V. (1941). Anthropometry: a practicalcourse. M.: Uchpedgiz.

[2] Chancy, U., Sequeira, P. S., \& Jain, J. (2015). Ethnic variation of selected dental traits in Coorg. Journal of Forensic Dental Sciences, 7(3), 180. doi: 10.4103/0975-1475.172422

[3] Daraze, A., Delatte, M., Bou Saba, S., \& Majzoub, Z. (2017). Craniofacial characteristics in the sagittal dimension: $A$ cephalometric study in Lebanese young adults. International Orthodontics, 15(1), 114-130. doi: 10.1016/j.ortho.2016.12.001

[4] Dmitriev, M. O. (2017). Relationships of linear parameters of the upper jaw with the characteristics of the closing plane, the position of the teeth and the profile of the soft tissues of the face in boys and girls. Biomedical and Biosocial Anthropology, 28, 95-101.

[5] Eigbobo, J. O., Sote, E. O., \& Oredugba, F. A. (2011). Variations of direct reliable medium strength, $4.2 \%$ of direct unreliable medium strength, $0.7 \%$ of inverse reliable medium strength and $0.7 \%$ of inverse unreliable medium strength); with second teeth 9 correlations out of 288 possible $(0.7 \%$ of direct reliable medium strength, $1.0 \%$ of direct unreliable medium strength, $0.3 \%$ of inverse reliable medium strength and $1.0 \%$ of inverse unreliable medium strength); with the height of the teeth, their crowns and the length of the roots 21 correlations out of 288 possible (4.5\% of direct reliable medium strength and $2.8 \%$ of direct unreliable medium strength); with vestibular-lingual and mesio-distal dimensions 17 correlations out of 288 possible $(0.7 \%$ of direct reliable medium strength, $2.4 \%$ of direct unreliable medium force, $1.0 \%$ of inverse reliable medium force and $1.7 \%$ of inverse unreliable medium force).

Our results of qualitative and quantitative analysis of correlations differ from the results obtained in a similar sample of men in the northern region of Ukraine by V.O. Orlovsky [14] for premolars.

The conducted correlation studies demonstrate the population specificity of the features of the dental system, which vary significantly depending on ethnicity and climatic and geographical conditions of human habitation.

\section{Conclusions}

Peculiarities of correlations of linear sizes of molars with cephalometric indicators of brain and facial skull of practically healthy men of the northern region of Ukraine are established. With indicators of the brain skull - multiple, only direct reliable $(r=0.35-0.57)$ and unreliable $(r=0.30$ $0.35)$ of medium strength correlation $(18.7 \%$ in the upper jaw, of which the relative majority with vestibular-lingual and mesio-distal size and $24.1 \%$ on the lower jaw, of which the relative majority with the height of the teeth, their crowns and the length of the roots of the teeth); with facial skull indices - mostly direct reliable $(r=0.35-0.51)$ and unreliable medium strength $(r=0.30-0.37)$ correlations, in most cases of a single nature $(6.0 \%$ on the upper jaw and $6.6 \%$ on the lower jaw, almost evenly with the height of the teeth, their crowns, the length of the roots, vestibular-lingual and mesio-distal dimensions).

of crown dimensions of permanent dentitions in a selected population of Nigerian children. Nig. Q. J. Hosp. Med., 21(2), 163-168. PMID: 21913517

[6] Furtado, G. C., Furtado, A., Abu El Haje, O., Butignon, L. E., Pesqueira, A. A., \& Paranhos, L. R. (2014). Relationship between the morphology of the maxillary central incisor and horizontal and vertical measurements of the face. Indian. $J$. Dent. Res., 25(2), 178-183. doi: 10.4103/0970-9290.135914 [7] Gayvoronskiy, I. V., Gayvoronskiy, A. I., Gayvoronskaya, M. G., Dubovik, E. I., Kevorkova, S. A., Koltunov, A. V., ... Schanikova, A. S. (2008). Craniometric correlations of the dentoalveolar system, temporomandibular joint and facial skull in an adult. Morphology, 133(2), 29.

[8] Glushak, A. A., Gunas, I. V., Lisnichuk, N. E., \& Samoylenko, A. V. (2015). The vertical sizes of teeth in adolescents of Podillia 
with an orthognathic bite depending on the shape of the head and type of face. Biology wa Tibbiyot Muammolari, 2(83), 28-37.

[9] Habib, S. R., Shiddi, I. A., Al-Sufyani, M. D., \& Althobaiti, F. A. (2015). Relationship and inter observer agreement of tooth and face forms in a Saudi subpopulation. J. Coll. Physicians Surg. Pak., 25(4), 276-280. doi: 04.2015/JCPSP.276280

[10] Isa, Z. M., Tawfiq, O. F., Noor, N. M., Shamsudheen, M. I., \& Rijal, O. M. (2010). Regression methods to investigate the relationship between facial measurements and widths of the maxillary anterior teeth. J. Prosthet. Dent., 103(3), 182-188. doi: 10.1016/S0022-3913(10)60028-5

[11] Kamiloglu, B., \& Kelahmet, U. (2014). Prevalence of impacted and transmigrated canine teeth in a Cypriote orthodontic population in the Northern Cyprus area. BMC Research Notes, 7(1), 346. doi: 10.1186/1756-0500-7-346

[12] Lombardo, L., Coppola, P., \& Siciliani, G. (2015). Comparison of dental and alveolar arch forms between different ethnic groups. International Orthodontics, 13(4), 462-488. doi: 10.1016/j.ortho.2015.09.013

[13] Niazi, M., Najmi, Y., \& Qadri, M. M. (2016). Frequency of cusp of carabelli in orthodontic patients reporting to Islamabad Dental Hospital. Pakistan Orthodontic Journal, 8(2), 85-88.

[14] Orlovskiy, V. O. (2017). Features relations computed tomography sizes of premolar teeth with cephalometric indicators of practically healthy men from northern and southern regions of Ukraine. Biomedical and Biosocial Anthropology, 29, 73-77.
[15] Padilla, M., Tello, L., Moreno, F., Osorio, J. C., \& Bedoya, A. (2013). Analysis of dental arch dimensions in three colombian ethnic groups. Int. J. Morphol., 31(1), 100-106. doi: http:// dx.doi.org/10.4067/S0717-95022013000100015

[16] Proffit, U. R. (translation from English; Ed. L.S. Persina) (2015). Modern Orthodontics. M.: Medpress-inform. ISBN: 978-500030-236-1

[17] Saidaloevich, M. S., Dusmukhamedov, M. Z., \& Murtazaev, S. S. (2015). Ethnic aspects of orthognathic bite. European Science Review, (7-8), 80-85.

[18] Samusev, R. P., Krayushkin, A. I., \& Dmitrienko, S. V. (Ed. M.R. Sapin) (2002). Basics of clinical morphology of teeth: a tutorial. M: OOO "Publishing House ONIX21 vek": OOO "World and Education". ISBN: 5-329-00426-8.

[19] Tabachnick, G. B., \& Fidell, L. S. (2014). Using multivariate statistics (6th ed.). Edinburgh: Pearson Education Limited.

[20] Vorobets, A. B., Hasiuk, P. A., \& Rosolovska, S. O. (2015). Aspects of caries development in fissure pits of molars in the gender aspect. Clinical Dentistry, (3-4), 132-133.

[21] Vorobets, A. B., Hasiuk, P. A., \& Rosolovska, S. O. (2016). Gender differences in odontometric parameters of crowns of molars. World of Medicine and Biology, 4(58), 16-19.

[22] Zhao, Q., Li, N., \& Cao, J. (2015). Morphological features of maxillary anterior teeth in a sample of Chinese population. Homo, 66(5), 448-454. doi: 10.1016/j.jchb.2015.06.001

[23] Zubov, A. A. (1968). Odontology. Methodology of anthropological research. M.: Science.

\section{КОРЕЛЯЦІЇ ЛІНІЙНИХ РОЗМІРІВ ВЕЛИКИХ КУТНІХ ЗУБІВ ІЗ КЕФАЛОМЕТРИЧНИМИ ПОКАЗНИКАМИ ПРАКТИЧНО ЗДОРОВИХ ЧОЛОВІКІВ ПІВНІЧНОГО РЕГІОНУ УКРАЇНИ \\ Поліщук С.С., Кочюра О.О., Дмітрієв М.О., Орловський В.О., Попов М.В.}

Питання взаємозв'язку метричних характеристик черепних структур і одонтометричних показників, та можливість побудови на їх основі моделей, що в подальшому можуть знайти своє практичне застосування в стоматологічній практиці, залишаються актуальною проблемою сучасної медичної науки. Мета дослідження - встановити особливості зв'язків лінійних розмірів великих кутніх зубів з кефалометричними показниками мозкового та лицевого черепа практично здорових чоловіків північного регіону України. У 32 практично здорових мешканців північного регіону України (з Київської, Житомирської, Сумської та Чернігівської областей) проведено комп'ютерну томографрію з наступною одонтометрією великих кутніх зубів та кефралометрію. Оцінку кореляцій між одонтометричними і кефралометричними показниками проводили з використанням непараметричної статистики Спірмена у ліцензійному пакеті "Statistica 6.1". Встановлені особливості зв'язків досліджених характеристик великих кутніх зубів із показниками мозкового черепу - множинні, лише прямі достовірні $(r=0,35-0,57)$ та недостовірні $(r=0,30-0,35)$ середньої сили кореляції $(18,7 \%$ на верхній щелепі, з яких відносна більшість із присінковоязиковими і мезіо-дистальними розмірами та 24,1\% на нижній щелепі, з яких відносна більшість із висотою зубів, їх коронок і довжиною коренів зубів); а також із показниками лицьового черепу - переважно прямі достовірні $(r=0,35-0,51)$ та недостовірні середньої сили $(r=0,30-0,37)$ зв'язки, у більшості випадків поодинокого характеру (6,0\% на верхній щелепі та 6,6\% на нижній щелепі, майже рівномірно з висотою зубів, їх коронок, довжиною коренів, присінково-язиковими та мезіо-дистальними розмірами). Результати кореляційного аналізу краніофаціальних структур необхідні для коректної побудови математичних моделей, які в подальшому можуть знайти своє практичне застосування в стоматологічній практиці та в судовій медицині. Ключові слова: кореляції, кефралометрія, одонтометрія, адміністративно-територіальні регіони України.

\section{КОРРЕЛЯЦИИ ЛИНЕЙНЫХ РАЗМЕРОВ БОЛЬШИХ КОРЕННЫХ ЗУБОВ С КЕФАЛОМЕТРИЧЕСКИМИ ПОКАЗАТЕЛЯМИ ПРАКТИЧЕСКИ ЗДОРОВЫХ МУЖЧИН СЕВЕРНОГО РЕГИОНА УКРАИНЫ \\ Полищук С.С., Коцюра О.А., Дмитриев Н.А., Орловский В.А., Попов М.В.}

Вопрос взаимосвязи метрических характеристик черепных структур и одонтометрических показателей и возможность построения на их основе моделей, которые в дальнейшем могут найти свое практическое применение в стоматологической практике, остаются актуальной проблемой современной медицинской науки. Цель исследования - установить особенности связей линейных размеров больших коренных зубов с кефралометрическими показателями мозгового и лицевого черепа практически здоровых мужчин северного региона Украины. Для 32 практически здоровых жителей северного региона Украины (жители Киевской, Житомирской, Сумской и Черниговской областей) проведена компьютерная томография $c$ последующей одонтометрией больших коренных зубов и кефралометрия. Оценку корреляций между одонтометрическими и кефралометрическими показателями проводили с использованием непараметрической статистики Спирмена в лицензионном пакете "Statistica 6.1". Установлены особенности связей исследованных характеристик больших коренных зубов с показателями мозгового черепа - множественные, только прямые достоверные ( $r=0,35-0,57)$ и недостоверные $(r=0,30-0,35)$ средней силы корреляции $(18,7 \%$ на верхней челюсти, из которых относительное большинство с вестибуло- 
оральными и мезио-дистальными размерами и 24,1\% на нижней челюсти, из которых относительное большинство с высотой зубов, их коронок и длиной корней зубов); а так же с показателями лицевого черепа - преимущественно прямые достоверные $(r=0,35-0,51)$ и недостоверные средней силы $(r=0,30-0,37)$ связи, в большинстве случаев одиночного характера (6,0\% на верхней челюсти и 6,6\% на нижней челюсти, почти равномерно с высотой зубов, их коронок, длиной корней, преддверно-языковыми и мезио-дистальными размерами). Результаты корреляционного анализа краниофациальных структур необходимы для корректного построения математических моделей, которые в дальнейшем могут найти свое практическое применение в стоматологической практике и в судебной медицине.

Ключевые слова: корреляции, кефалометрия, одонтометрия, административно-территориальные регионы Украины. 